\title{
The Impact of COVID-19 on Gold Price Volatility
}

Submitted 10/07/20, $1^{\text {st }}$ revision 29/08/20, $2^{\text {nd }}$ revision 17/09/20, accepted 30/09/20

\begin{abstract}
Ibrahim Yousef $^{1}$, Esam Shehadeh ${ }^{2}$
Abstract:

Purpose: This article investigates the implications of the spread of COVID-19 on gold spot prices.

Design/Methodology/Approach: We use GARCH and GJR-GARCH models based on daily gold returns over the period 2012-2020 to analyze the impact of the coronavirus on the volatility of gold returns.
\end{abstract}

Findings: We find a positive correlation between the increasing number of global coronavirus cases and increases in gold price. Using GARCH and GJR-GARCH models, we find a significant positive impact of COVID-19 on the conditional variance equation, indicating that the coronavirus may indeed increase the volatility of gold returns. This relates to the fact that the spread of the virus increases uncertainty with regard to the future of economic and financial markets, causing the demand for gold to increase and in turn pushing prices upwards, a trend which may be likely to continue until a vaccine or other treatments begin to stabilize the global economic outlook.

Practical Implications: The issue of volatility is of significant concern to both investors and policymakers who base decisions on the relative stability of both individual financial markets and the world economy. Furthermore, volatility estimation is an essential factor in many models and has broad application to the market risk management practices of firms. Finally, understanding the volatility of the gold market is crucial for any analysis of current and future expectations regarding the risks associated with coronavirus which apply to global markets.

Originality/Value: The lockdown restrictions which have been widely implemented across the globe to curb the spread of the virus have included travel prohibitions and border closures, stay-at-home and work-from-home orders, and extensive business closures, all causing immense fallout for the global economy. In the current study, we analyze for the first time the impact of the coronavirus on gold spot prices by examining their correlation with the number of cumulative global cases and daily new cases.

Keywords: Coronavirus, gold price, gold volatility, GARCH models.

JEL Code: C52, G15.

Paper Type: Research Paper.

ISSN: 2241-4754, H index 10, Q3.

\footnotetext{
${ }^{1}$ Department of Banking and Finance, Faculty of Administrative \& Financial Sciences, University of Petra, Amman, Jordan. Email: iyousef@uop.edu.jo;

${ }^{2}$ Department of Accounting, Faculty of Administrative \& Financial Sciences, University of Petra, Amman, Jordan. Email: esam.shehadeh@uop.edu.jo
} 


\section{Introduction}

The coronavirus (COVID-19) pandemic began in the city of Wuhan in the Hubei Province of China at the end of December 2019. While the fatality rate of this virus is considerably lower than that of the severe acute respiratory syndrome (SARS) outbreak in 2002-2003 or that of the Spanish Flu pandemic of 1919, COVID-19 is highly contagious and has triggered a great deal of worldwide economic and financial uncertainty (Zeren and Hizarci, 2020; Khan et al., 2020). The spread of the virus and globally enforced lockdowns have negatively impacted the overall demand and created significant short-term food price volatility (Albulescu, 2020). The fear engendered by the virus has put substantial stress on financial markets, where price volatility has been increasing continuously. In terms of the virus's human tragedy, the heaviest toll is seen in the United States and Europe.

At the time of writing this paper (05-May-2020), the number of global confirmed cases has exceeded 3.5 million, with more than 200,000 confirmed deaths. The highest virus-related death tolls have been recorded in the U.S., the UK, Italy, and Spain, with over a thousand deaths reported per day during the virus's peak in these countries. Healthcare systems have been strained to capacity and beyond, and there have been significant shortages in protective equipment for health workers and ventilators for patients, which has undoubtedly increased both infection and mortality rates.

The lockdown restrictions widely implemented across the globe to curb the spread of the virus have included travel prohibitions and border closures, stay-at-home and work-from-home orders, and extensive business closures, all causing immense fallout for the global economy. The still-early data assessing these measures' impact reveals that economic conditions have deteriorated dramatically since January of this year. For example, it was reported that in the single week ending 21-March, 3.3 million Americans applied for unemployment benefits, with this number doubling a week later to 6.6 million applications in a seven-day period. Before the current crisis, the highest number of unemployment applications made in the U.S. in a single week had been 695,000 in the year 1982. Furthermore, global stock markets lost nearly a third of their value in the six-week period between 20-February and the end of March (FitzGerald et al., 2020).

The measures rolled out to combat the coronavirus resemble those who have been historically implemented during times of war around the world. According to OECD estimates, such measures are likely to decrease economic output by $20-25 \%$. As rising market volatility has increasingly led investors to pursue haven assets, the U.S. dollar has seen itself strengthened in the global exchange market. However, during times of economic uncertainty and instability, investors often turn to gold as a haven. Gold is a liquid, counter-cyclical asset, and a long-term store of value, which can thus help investors meet the core objectives of safety, liquidity, and return. Thus, gold prices have increased from $\$ 1,517.3$ to $\$ 1,712.39$ an ounce (by 12.85\%) so far 
this year (from 01-Jan to 01-May 2020) and seem likely to go higher still. This means they are outperforming the American dollar.

In the current study, we analyze for the first time the impact of the coronavirus on gold spot prices by examining their correlation with the number of cumulative global cases and daily new cases. We find a positive correlation between these variables, indicating that the number of coronavirus cases has positively impacted gold prices. We attribute this correlation to the increased economic uncertainty caused by the spread of the virus. In addition, the global recession likely to be caused by the COVID-19 pandemic may mean investors will continue to seek refuge in gold for some time to come. Therefore, the demand for gold may continue to increase, simultaneously pushing its price upward until a vaccine or other virus treatments appear to stabilize the global economic outlook (Grima et al., 2020).

In addition, we use GARCH and GJR-GARCH models based on daily gold returns over the period 2012-2020 to analyze the impact of the coronavirus on the volatility of gold returns. We find a significant positive impact of COVID-19 on the conditional variance equation, which indicates that the virus has increased the volatility of these returns. Furthermore, we observe that bad news tends to increase the volatility of gold returns more than good news, and it thus makes sense that increasing the number of coronavirus cases (a bad news indicator) would increase the volatility of gold returns. We predict that because experts still struggle to both a) estimate the full eventual impact of the virus on the global economy and b) predict when a vaccine or other effective treatment might become widely available, gold prices will remain sensitive to market volatility for some time to come. Moreover, given the highly integrated nature of the global economy and the tendency for lookalike markets and currencies to move in tandem, further increases in the desire for gold and gold price volatility seem likely.

The results of this study will be of widespread interest for several reasons:

1. Volatility is of significant concern to both investors and policymakers who base decisions on the relative stability of both individual financial markets and the world economy.

2. Volatility estimation is an essential factor in many models and broadens firms' market risk management practices.

3. Understanding the gold market volatility is crucial for any analysis of current and future expectations regarding the risks associated with coronavirus, which apply to global markets.

The rest of this paper is organized as follows. Section 2 discusses historical gold prices and the data on a) cumulative coronavirus cases, b) daily new cases, c) the growth rate in daily new cases, and d) in the total number of cases. Section 3 discusses the study's data collection procedures and methodology. Section 4 presents the results of the data analysis, and Section 5 concludes. 


\section{Gold Prices and COVID-19}

Figure 1 presents the data for total global coronavirus cases and daily new cases for March and April 2020. There was a substantial increase in the number of new cases during March. On 01-Mar-2020, the number of new cases recorded globally was 1,811 . However, by 31 -Mar, this number had increased to 72,736 new cases recorded in a single day, yielding a per-day average for March of 21,467 new cases. In April, on the other hand, the trend is much more stable. The global total of the number of new cases recorded on 01-Apr was 72,849, compared with 71,764 new cases recorded on 30-Apr, and an average of 77,985 daily new cases over the course of the month. It can be seen in Figures 1 and 2 that the trends for both daily new cases and the growth rate in new cases were much more stable in April of 2020 than in March.

Figure 1. Number of new cases and total number of cases.

Figure 2. Daily growth rate in number of new cases and total number of cases.

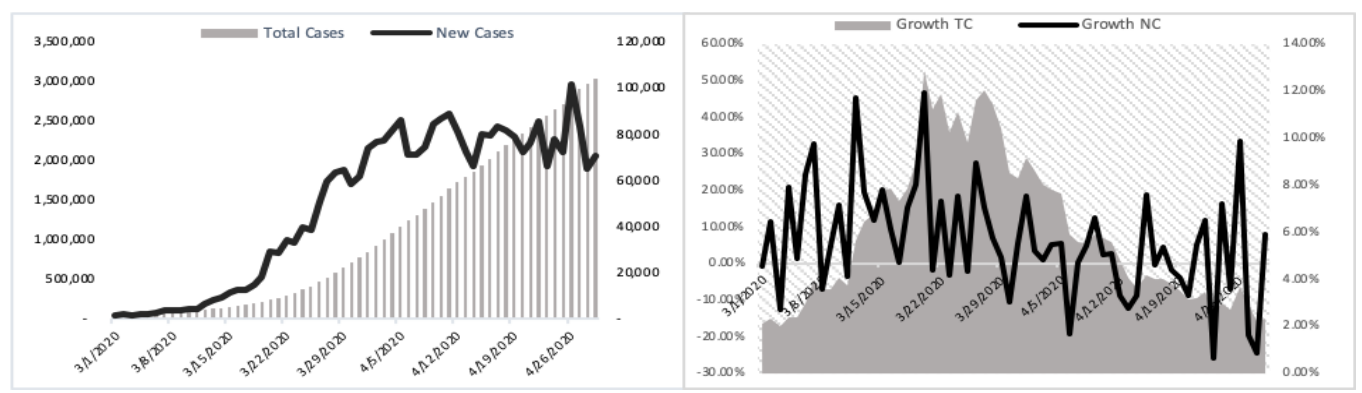

Source: Authors.

Figure 2 presents the data for both the growth rate in daily new cases and the cumulative number of cases. As noted above, greater stability can be observed in the growth rate of new cases for April compared with March; specifically, the average growth rate in daily new cases for March was $11.27 \%$ (-20.50\% minimum rate and 44.76\% maximum rate), while in April the average growth rate was $0.73 \%(-22.88 \%$ minimum rate and $23.32 \%$ maximum rate).

Figure 3 presents the data for the average weekly number of new cases and the growth rate in new cases. Although a clear increase is visible in the number of new cases over the last three months, the average weekly growth rate trend is declining with a coefficient of -158.3 . Furthermore, Figure 3 shows relative stability in the average number of global weekly new cases. 
Figure 3. Average weekly number of new growth rate in number of new cases.
Figure 4. Daily gold spot cases and prices in U\$

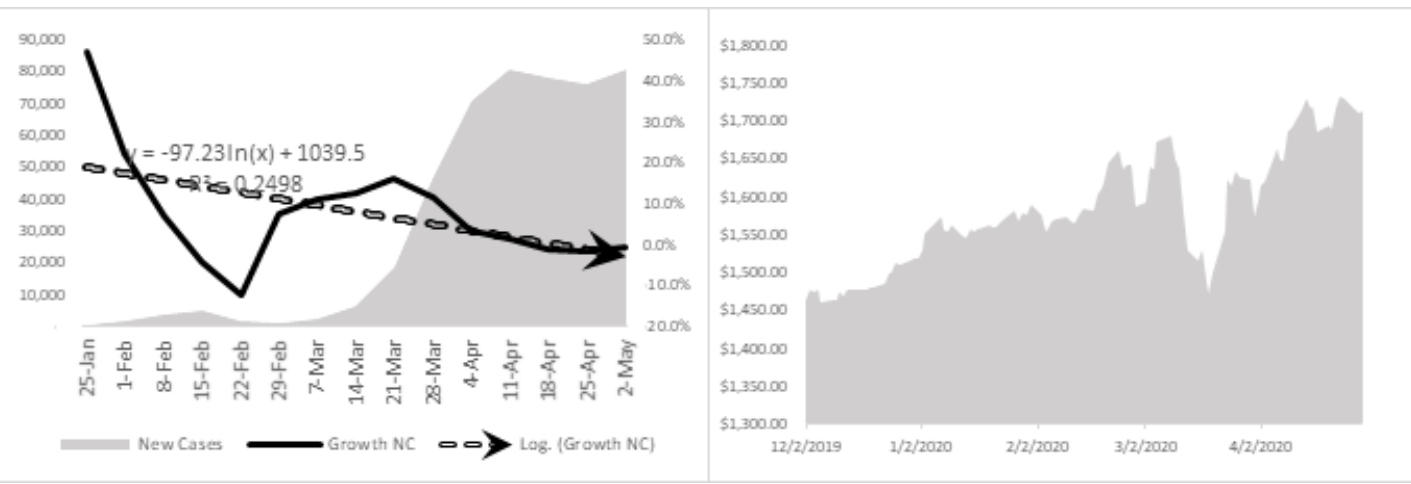

Source: Authors.

Figure 4 presents daily gold spot prices (in U.S. dollars) over the period 01-Jan to 01-May-2020. A clear increase in the gold price can be seen during the beginning of the coronavirus period (1-Jan to 9-Mar), from $\$ 1,517$ to $\$ 1,680$ an ounce. However, the price then began to decline, reaching a low of $\$ 1,471$ on 19-Mar. Shortly thereafter, it rebounded to reach a high of \$1,711 per ounce at the end of April.

\section{Methodology and Data Collection}

\subsection{Regression Model}

The total number of daily global new coronavirus cases and the cumulative number of cases were extracted from the daily situation reports released by the World Health Organization (WHO) beginning on 21-Jan-2020. Our sample thus covers the period 21-Jan to 1-May-2020 and includes 102 observations. In addition, the daily gold spot prices were collected from Thomson Reuters for the timeframe 2012-2020, which we divide into two periods of study. The first period, January to May 2020, is used in the regression analysis to assess the impact of COVID-19 on gold spot prices, while the second period, Jan-2012 to May-2020, is used in the GARCH model to analyze the impact of the virus on the volatility of gold returns.

Concerning the impact of coronavirus on gold spot prices from January to May 2020, the dependent variable is the daily gold spot price in U.S. dollars, and the independent variables are 1) the cumulative total number of daily cases and 2) the total number of new cases. The relevant equations can be written as follows:

$$
\begin{aligned}
& G S P_{t}=\beta_{0}+\beta_{1} \log T C_{t} \\
& G S P_{t}=\beta_{0}+\beta_{1} \operatorname{LogN} C_{t}
\end{aligned}
$$


where: GSP is the daily gold spot price in U.S. dollars, LogTC is the logarithm of the cumulative total number of daily new cases, and LogNC is the logarithm of the number of daily new cases.

\subsection{Volatility Models (GARCH Models)}

Since Engle originally introduced autoregressive conditional heteroscedasticity (ARCH) models in 1982, this method, along with its even more relevant cousin, the GARCH model (Bollerslev, 1986), become standard tools for assessing the volatility of financial variables. According to Sadorsky (2006), the model is extremely useful for observing heteroskedastic behavior or volatility clustering in financial markets without requiring higher-order models. Essentially, the model works by predicting variance in the current period by forming a weighted average of a) a long-term average, $b$ ) the variance forecasted in the previous period, and c) information about volatility observed in the previous period. In cases where a return is surprisingly large in either a positive or a negative direction for a given period, the trader will respond by increasing the variance estimate for the following period. The model also appropriately represents the volatility clustering often witnessed in financial returns data, where significant changes in returns tend to be followed by even more substantial changes (Trück, 2020; Yousef, 2020). In a $\operatorname{GARCH}(1,1)$ model, the conditional variance equation can be written as follows:

$h_{t}=\omega+\alpha_{1} \varepsilon_{t-1}^{2}+\beta_{1} h_{t-1}$

while the equation for the conditional mean is

$y_{t}=\mu+\varepsilon_{t}, \varepsilon_{t} \backsim N\left(0, h_{t}\right)$

where: $y_{t}$ is the conditional mean, $h_{t}$ is the conditional variance, $\mu$ and $\omega$ are constants, $\alpha_{1}$ is the ARCH term, $\beta_{1}$ is the GARCH term, and $\varepsilon_{\mathrm{t}}$ is the error term. A further extension of the ARCH and GARCH models is known as the GJR-GARCH model, which was developed by Glosten et al. (1993) and is useful for describing return asymmetry. Substantial negative returns are often followed by significantly increased volatility, such that the GJR models differentiate between positive and negative returns. The GJR model can be written as follows:

$h_{t}=\omega+\alpha_{1} \varepsilon_{t-1}^{2}+\beta_{1} h_{t-1}+\gamma_{i} I_{t-1} \varepsilon_{t-1}^{2}$

where: $\mathrm{I}_{t-1}$ is equal to one if $\varepsilon_{t}<0$, and zero otherwise. Obviously, $\varepsilon_{t-1}^{2}>0$, and $\varepsilon_{t-1}^{2}<0$ in this model will have different impacts on the conditional variance. If $\gamma \neq$ 0 , there is asymmetry in the model, while $\gamma>0$ means that the occurrence of bad news will increase volatility and provide evidence of a leverage effect. Finally, we add a dummy variable to the conditional variance equations (Equations 3 and 5) to analyze the impact of the coronavirus on gold volatility, as follows: 
$h_{t}=\omega+\alpha_{1} \varepsilon_{t-1}^{2}+\beta_{1} h_{t-1}+\gamma_{i} I_{t-1} \varepsilon_{t-1}^{2}+\delta_{1} \operatorname{COVID}_{1}$

where: COVID is a dummy variable equal to one for the coronavirus period (January to May 2020) and zero otherwise, and where the period of study for the GARCH models is Jan-2012 to May-2020. Furthermore, we use daily gold returns calculated as the first differences of natural logarithmic daily gold closing prices.

\section{Results of Data Analysis}

\subsection{Descriptive Statistics of Gold Daily Returns}

Table 1 presents the results of the summary statistics, including mean, median, minimum value, maximum value, standard deviation, coefficient of variation (C.V.), skewness, and kurtosis for both the gold price and daily gold log-returns for the observations 3-Jan-2012 to 1-May-2020 (2,167 valid daily observations). The mean and median of daily returns are positive, indicating that overall gold prices were increasing during the period in question. Specifically, the gold price at the start of the period (3-Jan-2012) was $\$ 1,603.69$ an ounce, while the closing price the last day of the period (01-May-2020) was $\$ 1,699.12$ per ounce. However, the magnitude of the average daily gold return $(0.004 \%)$ and the median return $(0.012 \%)$ are small compared to the standard deviation (0.924\%). The minimum price of gold (\$1,051.75/ounce) was seen on 17-Dec-2015, while the maximum value (\$1,790/ounce) occurred on 4-Oct-2012. On the other hand, the lowest daily return for gold $(-8.879 \%)$ occurred on 15-Apr-2013, while the largest $(4.69 \%)$ was seen on 24-Jun-2016, followed by the second largest (4.3\%) on 24-Mar-2020.

Table 1. Summary statistics using observations 2012-01-02 to 2020-04-29

\begin{tabular}{lllll}
\hline Gold & Mean & Median & Minimum & Maximum \\
\hline Price & 1345.2 & 1291.5 & 1051.7 & 1790.6 \\
Return & $0.004 \%$ & $0.012 \%$ & $-8.879 \%$ & $4.693 \%$ \\
\hline & Std. Dev. & C.V. & Skewness & Kurtosis \\
\hline Price & 172.85 & 0.12849 & 0.89413 & -0.20784 \\
Return & $0.924 \%$ & 221.31 & -0.55768 & 7.3016 \\
\hline
\end{tabular}

Source: Own study.

The large kurtosis value of 7.3 indicates that daily gold returns are subject to leptokurtic characteristics, revealing a peaked distribution. Further, the variable's skewness was -0.557 , and this negative value indicates that the distribution has a long-left tail. It is clear that the series distribution features significantly fatter tails than those of a normal distribution, and the Jarque and Bera (1980) test statistic supports this indication of non-normality, allowing rejection of the null hypothesis of normal distribution at all significance levels (Table 2). 
Table 2. Summary statistics for the Dickey-Fuller, ARCH effect and Jarque-Bera tests

\begin{tabular}{llll} 
Dickey-Fuller tests Statistic & & ARCH effect & Jarque-Bera \\
\hline Level & First Difference & LM & \\
$-0.996^{* * *}$ & $-13.508^{* * *}$ & $2137.03 * * *$ & $4926.02 * * *$ \\
\hline
\end{tabular}

Source: Own study.

In addition, we use the Augmented Dick Fuller (ADF) (1979) test to test for stationarity of the return series. The null hypothesis (H0) for this test is that the time series has a unit root, while $\mathrm{H} 1$ states that it is stationary. The results indicate that the return series gold prices can be considered stationary, and the null hypothesis is thus rejected.

Concerning testing for $\mathrm{ARCH}$ effects, the null hypothesis states that no ARCH is present while $\mathrm{H} 1$ states the opposite. According to this test results, the null hypothesis is rejected at a significance level of $1 \%$, and it can thus be concluded that ARCH effects are present. In other words, the variance is not constant, and the GARCH method should be used to model changing volatility and determine whether the coronavirus impacts return on the variables. The GARCH method's benefit is that it helps to explain the non-constant characteristics or behavior of the variance. Before turning to the GARCH model, however, we will first analyze the impact of COVID-19 on gold prices (equations 1 and 2).

\subsection{Results for the Impact of the Coronavirus on Gold Prices}

The results reveal a significant positive impact of the logarithm of the cumulative total number of daily cases and the logarithm of the number of new cases on daily gold prices, implying that gold prices will increase the global number of coronavirus cases increases. This can be attributed to the fact that the escalating spread of coronavirus correspondingly increases uncertainty about the future of financial markets and the global economy.

In such cases, most companies and investors seek out assets that negatively correlate with other assets during the crisis period. As discussed above, these are known as haven assets, and they are expected to gain value (or at least retain a stable one) during periods of economic downturn. Gold is such an asset; it can be argued that its value may be likely to continue increasing throughout the current period of economic instability, at least until a vaccine or other effective treatment is found, which can help stabilize the global economic outlook (Table 3).

Table 3. Summary statistics for the regression analysis.

\begin{tabular}{lllll}
\hline & OLS & OLS & HCM & HCM \\
\hline $\mathrm{R}^{2}$ & 0.426 & 0.299 & 0.432 & 0.395 \\
Adjusted $\mathrm{R}^{2}$ & 0.419 & 0.291 & 0.425 & 0.388 \\
$\mathrm{~F}(24,20528)$ & $63.136^{* * *}$ & $36.257^{* * *}$ & $64.606 * * *$ & $55.602 * * *$
\end{tabular}




\begin{tabular}{lllll} 
P-value(F) & 0.000 & 0.000 & 0.000 & 0.000 \\
\hline const & $1485^{* * *}$ & $1532^{* * *}$ & $1512^{* * *}$ & $1544^{* * *}$ \\
& 0.000 & 0.000 & 0.000 & 0.000 \\
LogTC & $26.306^{* * *}$ & & $18.852^{* * *}$ & \\
& 0.000 & & 0.000 & \\
LogNC & & $21.701^{* * *}$ & & $17.478^{* * *}$ \\
& & 0.000 & & 0.000 \\
\hline
\end{tabular}

Note: We use ordinary least squares (OLS) regression and heteroskedasticity-corrected model (HCM), where the dependent variable is the daily gold spot price in U.S. dollars (GSP), LogTC is the logarithm of the cumulative total number of daily new cases, and LogNC is the logarithm of the number of daily new cases.

Source: Own study.

This section aims to analyze the coronavirus's impact on daily gold spot prices over the period Jan-2020 to May-2020. The dependent variable is the daily gold spot in U.S. dollars, and the independent variables are 1) the logarithm of the cumulative total number of daily cases $(\log \mathrm{TC})$, and 2$)$ the logarithm of the number of new cases $(\operatorname{LogNC})$. The results are presented below in Table 3.

\subsection{Volatility Measures Using GARCH Models}

The GARCH model is used in this study to determine whether the coronavirus is impacting the volatility of gold returns. Since several previous studies have noted this specification's appropriateness for studying time series, we analyze the estimation of the generalized model to the case where both $\mathrm{p}$ and $\mathrm{q}$ are set to 1 . The values $(\mathrm{p}<=2, \mathrm{q}<=2)$ are sufficient to model volatility in most cases and provide a sufficient trade-off between flexibility and parsimony (Knight and Satchell, 1998). Franses and Van Dijk (1996) and Gokcan (2000) have also demonstrated that models with a small lag, such as the $\operatorname{GARCH}(1,1)$, are suitable for cases of changing variance, and Brooks (2002) asserts that the lag order model $(1,1)$ is capable of capturing all of the volatility clustering that may be present within the data. Furthermore, the GJR-GARCH(1,1) is thought to be the most successful model insofar as it exhibits the smallest value while still adhering to restrictions such as that of non-negativity for the symmetric GARCH. These models are estimated using quasi-maximum likelihood and assuming a Gaussian normal distribution. The results are presented in Tables 4 and 5 below.

The GARCH(1,1) model is used to estimate stock market volatility for daily gold returns. We use dummy variables in this analysis to indicate whether the coronavirus is influencing gold returns or not, where 1 represents the coronavirus period, i.e., Jan-2020 to May-2020 and 0 indicates all other dates. It can be seen in Table 4 that the ARCH effects $(\alpha 1)$ are significant and positive, which implies substantial volatility clustering. Similarly, the GARCH effect $(\beta 1)$ is also positive and significant, indicating persistent volatility. Thus, the variance equation suggests that the COVID-19 virus is playing a significant role in the volatility of gold returns. 
Table 4. Summary statistics for $\operatorname{GARCH}(1,1)$.

\begin{tabular}{llll|llll}
\hline & coefficient & $\mathrm{p}$-value & & \multicolumn{2}{l}{ coefficient } & $\mathrm{p}$-value \\
\hline $\begin{array}{l}\text { Conditional mean equation } \\
\text { const }\end{array}$ & 0.0037 & 0.829 & & 0.0042 & 0.810 & \\
\hline $\begin{array}{l}\text { Conditional variance equation } \\
\text { omega }\end{array}$ & 0.0095 & 0.000 & $* * *$ & 0.0067 & 0.000 & $* * *$ \\
alpha & 0.043 & 0.000 & $* * *$ & 0.035 & 0.000 & $* * *$ \\
beta & 0.947 & 0.000 & $* * *$ & 0.957 & 0.000 & $* * *$ \\
COVID-19 & & & & $\mathbf{0 . 0 2 1 9}$ & $\mathbf{0 . 0 0 3}$ & $* * *$ \\
\hline Llik: & 7217 & & & 7221 & & \\
AIC: & -14427 & & & -14433 & & \\
\hline
\end{tabular}

Source: Own study.

Table 5 presents the results of the GJR-GARCH(1,1) model analyzing the impact of the coronavirus on the volatility of gold returns for the period Jan-2012 to May2020. First, alpha and beta are significant in the GJR model, indicating persistent volatility and significant volatility clustering. For the coronavirus variable, the results are positive and significant in the conditional variance equation, indicating that the virus is increasing the volatility in gold returns.

Table 5. Summary statistics for GJR-GARCH(1,1).

\begin{tabular}{|c|c|c|c|c|}
\hline & coefficient & p-value & coefficient & p-value \\
\hline \multicolumn{5}{|c|}{ Conditional mean equation } \\
\hline const & $7.19 \mathrm{E}-07$ & 0.997 & $-8.77 \mathrm{E}-07$ & 0.996 \\
\hline \multicolumn{5}{|c|}{ Conditional variance equation } \\
\hline omega & 0.0067 & $0.000 * * *$ & 0.0067 & $0.000 * * *$ \\
\hline alpha & 0.040 & $0.000 * * *$ & 0.032 & $0.000 * * *$ \\
\hline gamma & 0.085 & $0.021 * *$ & 0.112 & $0.004 * * *$ \\
\hline beta & 0.949 & $0.000 * * *$ & 0.959 & $0.000 * * *$ \\
\hline COVID-19 & & & 0.0223 & $0.001 * * *$ \\
\hline Llik: & 7218 & & 7223 & \\
\hline AIC: & -14427 & & -14434 & \\
\hline
\end{tabular}

Source: Own study.

Moreover, the results presented in Table 5 show that the gamma measuring leverage effect has a significant positive impact, which implies that negative shocks will lead to greater increases in future volatility than would positively shocks of the same magnitude. In other words, bad news tends to increase the volatility of gold returns to a greater extent than good news. Therefore, increases in the number of coronavirus cases (i.e., a bad news indicator) will increase gold returns volatility. This supports our findings in the previous section. The continued lack of knowledge about the virus and corresponding difficulty faced by economists to estimate its full eventual economic impact may mean that gold prices are likely to remain subject to market volatility for some time to come. Moreover, because the global economy has become increasingly integrated in recent decades and many look-alike markets and 
currencies tend to move in tandem, there is an increased demand for safe haven assets, such as gold, in uncertain scenarios like the current coronavirus crisis, and such scenarios are known to increase gold price volatility.

\section{Conclusion}

The outbreak of the COVID-19 virus has shaken financial markets. So far, confirmed infections have reached 3.3 million and have been reported in at least 200 countries, resulting in more than 250,000 deaths. The continued spread of the virus and the measures most governments have been forced to take to curb its proliferation have created widespread fear that global economic growth will plummet, and many investors are thus moving away from the stock market and seeking instead to purchase safe-haven assets such as gold.

The U.S. government responded to the situation in mid-March by slashing interest rates in what constitutes the first emergency cut since the 2008 crisis, indicating that officials expect the virus to have a significant negative impact on the world economy. The general uncertainty created by the situation leads to many important questions, one of which concerns the virus's impact on the gold market. To answer this question, we analyze for the first time the impact of COVID-19 on gold spot prices and find that the increasing number of global coronavirus cases correlates to an increase in gold prices, which can be attributed to the uncertainty about future economic conditions created by the virus and the fact that gold is considered a safe investment in uncertain times. We also find a significant positive impact of COVID19 on the conditional variance equation, implying that the virus is also increasing the volatility of gold returns.

As the ongoing situation increases the global demand for gold, both gold prices and the volatility of returns will also increase. This is because of gold's status as a 'safe have n' asset in times of economic turmoil, i.e., it tends to be uncorrelated or even negatively correlated with other types of assets, which tend to lose value in such periods. This quality means that gold investment is an important diversification asset in portfolios, and similar spikes in gold prices and returns volatility have been witnessed in previous crises, while the overall gold market remained relatively stable.

\section{References:}

Albulescu, C. 2020. Coronavirus and oil price crash: A note. arXiv preprint arXiv:2003.06184.

Bollerslev, T. 1986. Generalized autoregressive conditional heteroskedasticity. Journal of econometrics, 31(3), 307-327.

Engle, R.F. 1982. Autoregressive conditional heteroscedasticity with estimates of the variance of United Kingdom inflation. Econometrica: Journal of the Econometric Society, 987-1007. 
FitzGerald, A., Kwiatkowski, K., Singer, V., Smit, S. 2020. An instant economic crisis: How deep and how long? McKinsey.

Franses, P.H., Van Dijk, D. 1996. Forecasting stock market volatility using (non-linear) Garch models. Journal of Forecasting, 15(3), 229-235.

Glosten, L.R., Jagannathan, R., Runkle, D.E. 1993. On the relation between the expected value and the volatility of the nominal excess return on stocks. The journal of finance, 48(5), 1779-1801.

Gokcan, S. 2000. Forecasting volatility of emerging stock markets: linear versus non-linear GARCH models. Journal of forecasting, 19(6), 499-504.

Grima, S., Dalli Gonzi, R., Thalassinos, I.E. 2020. The Impact of COVID-19 on Malta and its Economy and Sustainable Strategies. Available at SSRN: https://ssrn.com/abstract=3644833 or http://dx.doi.org/10.2139/ssrn.3644833.

Jarque, C.M., Bera, A.K. 1980. Efficient tests for normality, homoscedasticity and serial independence of regression residuals. Economics letters, 6(3), 255-259.

Khan, S., Rabbani, R.M., Thalassinos, I.E., Atif, M. 2020. Corona Virus Pandemic Paving Ways to Next Generation of Learning and Teaching: Futuristic Cloud Based Educational Model. Available at SSRN: https://ssrn.com/abstract=3669832.

Sadorsky, P. 2006. Modelling and forecasting petroleum futures volatility. Energy Economics, 28(4), 467-488.

Trück, S. 2020. Modelling and forecasting volatility in the gold market. International Journal of Banking and Finance, 9(1), 48-80.

Yousef, I. 2020. Spillover of COVID-19: Impact on Stock Market Volatility. International Journal of Psychosocial Rehabilitation, 24(06), 18069-18081.

Zeren, F., Hizarci, A. 2020. The Impact of COVID-19 Coronavirus on Stock Markets: Evidence from Selected Countries. Muhasebe ve Finans İncelemeleri Dergisi, 3(1), 78-84. 